\title{
An Optimization Method on Task Distribution at Dynamic Enterprise Alliance
}

\author{
Tie-jiang Yang \\ Inst. 365, North western Poly tech. Univ., Xi'an, China
}

Keywords: Enterprise alliance, Task distribution, Threshold.

\begin{abstract}
This paper analyses the needs of task distribution for dynamic enterprise alliance, and then proposes a fuzzy algorithm which gathers the tasks from enterprise to subprojects in alliance. It assigns values to the degrees of interactions of the task modules, and creates each adjacency matrix. It then performs fuzzy aggregation to the adjacency matrices by setting different thresholds. By combining the aggregation degree in the tasks modules with the separation degree between the modules, setting it as judge standard, and then, the values of the fitness function at different thresholds can be calculated. It finally obtains the best scheme of tasks modules distribution by using the threshold corresponding to the maximum fitness function.
\end{abstract}

\section{Introduction}

In order to take full advantage of the manufacturing and technical resources of each dynamic enterprise, the alliance has to complete the coordinated research project at the fastest speed and the lowest cost by using the information technology in a distributed condition. Aiming at improving the parallelism of task execution, facilitating the overall arrangement, clearing process management model, and improving the efficiency of task management, task decomposition should be conducted. Therefore, it becomes a key issue that how to decompose the given project into independent subprojects with reasonable granularity during the coordinated research of dynamic enterprise alliance (Figure. 1).

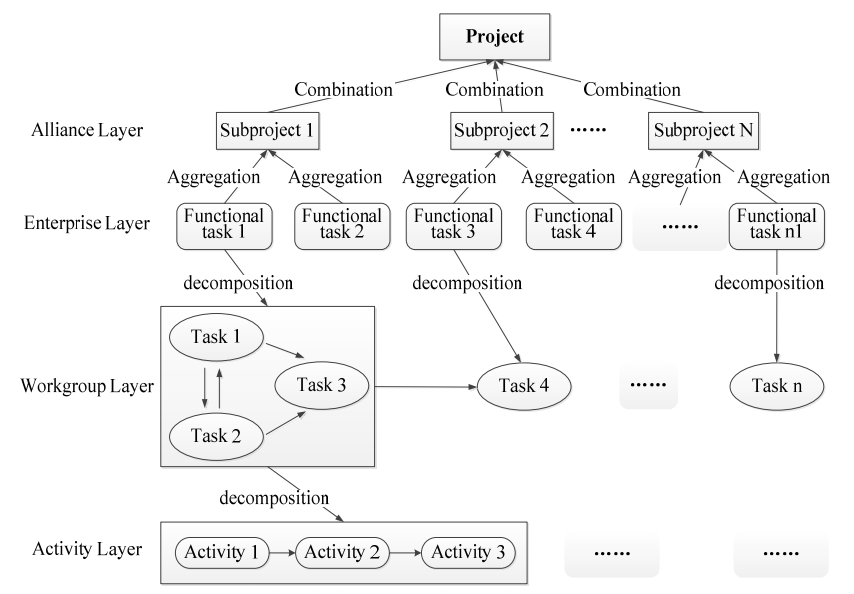

Figure.1: Task decomposition of dynamic enterprise alliance.

Conventional aggregation analysis decomposes the objects by their similarity [1], then divides each recognized object into a certain class strictly [2,3]. L. A. Zadeh proposed the theory of fuzzy sets $[4,5]$, and then called it fuzzy aggregation analysis. With this method, the fuzzy aggregation of functional task modules can be completed, and the interaction and the interaction cost of task modules between enterprises can be reduced. 


\section{Fuzzy Aggregation of Modules}

\section{Aggregation Condition}

The connection flow matrix between functional tasks modules is the superposition of the relationships of material, energy and information. Assuming that functional tasks are decomposed into $\mathrm{n}$ functional tasks modules $C=\left\{c_{1}, c_{2}, \ldots, c_{n}\right\} . m_{i j}$ represents the material connection between task module $i$ and $j . e_{i j}$ represents the energy connection between task module $i$ and $j . \alpha_{i j}$ represents the information connection between task module $i$ and $j$. The regulations of compact degree of material connection between modules are shown in Table 1.

Similarly, the assignment of the compact degree of information flow and energy flow between modules can be obtained. Assuming that $X$ represents the connection flow matrix, and $X_{i j}$ is its element, then $x_{i j}=m_{i j}+e_{i j}+\alpha_{i j}$.

Table 1: Assignments and meanings of the compact degree of the material flow between task modules.

\begin{tabular}{cc}
\hline Assignments & Meanings \\
\hline 0 & No material flows from task $C_{i}$ to $C_{i}$ \\
\hline 1 & Seldom materials flow from task $C_{i}$ to $C_{i}$ \\
\hline 3 & Less materials flow from task $C_{i}$ to $C_{j}$ \\
\hline 5 & More materials flow from task $C_{i}$ to $C_{j}$ \\
\hline 7 & Many materials flow from task $C_{i}$ to $C_{j}$ \\
\hline 9 & Much materials flow from task $C_{i}$ to $C_{i}$ \\
\hline $2,4,6,8$ & Intermediate values between above relevant judgments \\
\hline
\end{tabular}

\section{The Establishment of Matrix}

Since the data of matrix $\mathrm{X}$ has the same dimension, and in order to standardize the data, the similarity calibration can be performed and the fuzzy similar matrix R can be established, where $r_{i j}$ is the element. The fuzzy similar matrix $\mathrm{R}$ of matrix $\mathrm{X}$ can be obtained by the quantity product method.

$$
r_{i j}= \begin{cases}1, & i=j \\ \frac{1}{M} \sum_{k=1}^{m} x_{i k} \cdot x_{j k}, & i \neq j\end{cases}
$$

Where, $M$ is chosen as an appropriate value ${ }^{M=\max _{i \neq j}\left(\sum_{k=1}^{m} x_{i k} \cdot x_{j k}\right)}$, so that $0 \leq r_{i j} \leq 1$. Then we can get fuzzy similar matrix $R$.

In order to transform the fuzzy similar matrix $R$ into the transitive fuzzy equivalent matrix $R^{*}$, it is needed to perform the transitive closure on $R$.

\section{Fuzzy Aggregation}

In order to ascertain the ownership of an element in the fuzzy set, a threshold ${ }^{\alpha}$ should selected first in $\alpha \in[0,1]$. The fuzzy set in $U$ is 


$$
R^{*}=\left\{u_{i} \mid u_{i} \in U, 0 \leq \mu R\left(u_{i}\right) \leq 1\right\}
$$

When the value of $\alpha$ is selected, a corresponding common set can be obtained.

$$
R_{\alpha}=\{u \mid u \in U, \mu R(u) \geq \alpha\}
$$

Where, $R_{\alpha}$ is called as ${ }^{\alpha}$-level cut sets of $R^{*}$, and $\alpha$ is called as threshold of $R_{\alpha}$.

The characteristic function of $R_{\alpha}$ is

$$
\mathrm{C}_{R_{\alpha}}(u)= \begin{cases}1 & \mu R(u) \geq \alpha \\ 0 & \mu R(u)<\alpha\end{cases}
$$

At different thresholds $\alpha$, the aggregation analysis of fuzzy equivalent matrix $R^{*}$ can be conducted. And the aggregation results of different thresholds can be calculated by the following formula.

$$
r_{i j}= \begin{cases}0 & r_{i j}<\alpha \\ 1 & r_{i j} \geq \alpha\end{cases}
$$

\section{The Establishment of the Fitness Function and the Optimization of Task Distribution}

\section{The Establishment of the Fitness Function}

Suppose that there is a set of $q$ functional tasks $\mathrm{C}=\left\{c_{1}, c_{2}, \cdots, c_{q}\right\}$, and it is divided int ${ }^{p}$ modules $\mathrm{M}=\left\{M_{1}, M_{2}, \cdots, M_{P}\right\}, \bigcup_{i=1}^{p} M_{i}=C$, and $M_{i} \cap M_{j}=\varnothing$. There are $k_{i}$ functional tasks in each module, and $\sum_{i=1}^{p} k_{i}=q$.

Assuming that the functional tasks module $\mathrm{M}_{i}=\left\{\cdots, c_{b}, \cdots, c_{d}, \cdots\right\}$ has $n$ functional tasks, like $c_{b}$ and ${ }^{C_{d}}$. The aggregation degree of this module is expressed as:

$$
I C\left(M_{i}\right)=\left\{\begin{array}{l}
0, \quad n=1 \\
\frac{1}{k_{i}\left(k_{i}-1\right)} \sum_{b \in M_{i}, d \in M_{i}, b \neq d} \mathrm{c}_{b d}, \quad n \geq 2
\end{array}\right.
$$

The average aggregation degree is:

$$
\overline{I C}=\frac{1}{p} \sum_{i=1}^{p} I C\left(M_{i}\right)
$$

Obviously, the larger $I C\left(M_{i}\right)$, the more closely relationship between two units .Thus, the overall quality of the decomposition is higher.

According to the above definition, the aggregation degree between module ${ }^{M_{i}}$ and ${ }^{M_{j}}$ is:

$$
D\left(M_{i}, M_{j}\right)=\frac{1}{k_{i} k_{j}} \sum_{b \in M_{i}, d \in M_{j}} c_{b d}
$$


Obviously, the larger $D V\left(M_{i}, M_{j}\right)$, the smaller separation degree and the more closely relationship between module ${ }^{M_{i}}$ and ${ }^{M_{j}}$. Thus the overall quality is lower. So the average aggregation degree is:

$$
\overline{D V}=\frac{1}{p(p-1)} \sum_{i=1}^{p} \sum_{j=1}^{p} D V\left(M_{i}, M_{j}\right)
$$

Since the larger aggregation degree between task modules represents their separation degree is smaller, so the separation degree of module ${ }^{M_{i}}$ and $M_{j}$ is defined as:

$$
D C=\frac{1}{\overline{D V}}
$$

According to the analysis of the aggregation degree in the task modules and the separation degree between the tasks modules, the fitness function can be defined as:

$$
f(c)=\overline{I C} \cdot \overline{D C}=\frac{\overline{I C}}{\overline{D V}} \quad \overline{D V} \neq 0 .
$$

When $\overline{D V}=0$, all functional tasks are designated within one module, and all functional tasks appear in an enterprise. Since it is not conducive to the concurrent design of the tasks in the dynamic enterprise alliance, this kind of decomposition is generally not adopted.

\section{Optimization for Task Distribution}

By combining the aggregation degree in the tasks modules with the separation degree between the tasks modules, and setting it as judge standard, the values of the fitness function at different thresholds can be calculated. Finally, the best scheme of tasks modules decomposition is obtained by taking the threshold corresponding to the maximum fitness function as the best threshold.

\section{Case Study}

During the development of a certain aircraft, the leader of the dynamic enterprise alliance decomposed the main task into many functional tasks modules. Five of the modules are design of aircraft engine, fuel tank, hydraulic transmission system, power plant and back-fuselage, and they are denoted as $f_{1}, f_{2}, f_{3}, f_{4}$ and $f_{5}$ respectively. After the analysis and assignment of the flow of material, energy and information, the connection flow matrix between tasks modules is constructed as:

$f_{1}$
$f_{2}$
$f_{1}$
$f_{2}$
$f_{3}$
$f_{4}$
$f_{4}$$\left[\begin{array}{ccccc}0 & 10 & 7 & 8 & 7 \\ 7 & 0 & 6 & 12 & 21 \\ 7 & 4 & 0 & 2 & 19 \\ 8 & 3 & 12 & 0 & 25 \\ 7 & 3 & 6 & 12 & 0\end{array}\right]$

The fuzzy similarity matrix can be obtained by the quantity product method. 


$$
R=\left[\begin{array}{lcccc}
1 & 0.44 & 0.29 & 0.44 & 0.26 \\
0.44 & 1 & 0.72 & 1 & 0.35 \\
0.29 & 0.72 & 1 & 0.83 & 0.13 \\
0.44 & 1 & 0.83 & 1 & 0.21 \\
0.26 & 0.35 & 0.13 & 0.21 & 1
\end{array}\right]
$$

So the fuzzy equivalent matrix $t(R)=R^{4}=R^{*}$ is:

$$
R^{*}=\left[\begin{array}{lcccc}
1 & 0.44 & 0.44 & 0.44 & 0.35 \\
0.44 & 1 & 0.83 & 1 & 0.35 \\
0.44 & 0.83 & 1 & 0.83 & 0.35 \\
0.44 & 1 & 0.83 & 1 & 0.35 \\
0.35 & 0.35 & 0.35 & 0.35 & 1
\end{array}\right]
$$

Take the threshold $\lambda=1$, then

$$
R_{\lambda}^{*}=R_{1}^{*}=\left[\begin{array}{ccccc}
1 & 0 & 0 & 0 & 0 \\
0 & 1 & 0 & 1 & 0 \\
0 & 0 & 1 & 0 & 0 \\
0 & 1 & 0 & 1 & 0 \\
0 & 0 & 0 & 0 & 1
\end{array}\right]
$$

The decomposition result when $\lambda=1$ is $\left\{f_{1}\right\},\left\{f_{2}, f_{4}\right\},\left\{f_{3}\right\},\left\{f_{5}\right\}$.

The decomposition result when $\lambda=0.83$ is $\left\{f_{1}\right\},\left\{f_{2}, f_{3}, f_{4}\right\},\left\{f_{5}\right\}$.

The decomposition result when $\lambda=0.44$ is $\left\{f_{1}, f_{2}, f_{3}, f_{4}\right\},\left\{f_{5}\right\}$.

The decomposition result when $\lambda=0.35$ is $\left\{f_{1}, f_{2}, f_{3}, f_{4}, f_{5}\right\}$.

When $\lambda=1, M_{1}=\left\{f_{1}\right\}, M_{2}=\left\{f_{2}, f_{4}\right\}, M_{3}=\left\{f_{3}\right\}, M_{4}=\left\{f_{5}\right\}$

And from the fitness function, we can get:

$$
\begin{gathered}
I C\left(M_{1}\right)=I C\left(M_{3}\right)=I C\left(M_{4}\right)=0, \quad I C\left(M_{2}\right)=\frac{1}{2} \times 15 \\
\overline{I C}=\frac{1}{4} \times I C\left(M_{2}\right)=\frac{1}{4} \times \frac{1}{2} \times 15=\frac{15}{8} \\
\overline{D V}=\frac{1}{4 \times 3} \times\left(\frac{33}{2}+14+14+\frac{24}{2}+\frac{61}{2}+25\right)=\frac{87}{12} \\
f_{1}(c)=\frac{\overline{I C}}{\overline{D V}}=\frac{15}{8} \times \frac{12}{87} \approx 0.26 \\
\overline{I C}=\frac{1}{6} \times \frac{1}{3} \times 39=\frac{39}{18} \\
\frac{\mathrm{When}}{\overline{D V}}=\frac{1}{3 \times 2} \times\left(\frac{47}{3}+14+\frac{86}{3}\right)=\frac{175}{18}
\end{gathered}
$$




$$
f_{2}(c)=\frac{\overline{I C}}{\overline{D V}}=\frac{39}{18} \times \frac{18}{175} \approx 0.22
$$

When $\lambda=0.44, M_{1}=\left\{f_{1}, f_{2}, f_{3}, f_{4}\right\}, M_{2}=\left\{f_{5}\right\}$

$$
\begin{aligned}
& \overline{I C}=\frac{1}{2} \times \frac{1}{12} \times 86=\frac{86}{24} \\
& \overline{D V}=\frac{1}{2} \times \frac{1}{4} \times 100=\frac{100}{8} \\
& f_{3}(c)=\frac{\overline{I C}}{\overline{D V}}=\frac{86}{24} \times \frac{8}{100} \approx 0.29
\end{aligned}
$$

When $\lambda=0.35$, it has $M=\left\{f_{1}, f_{2}, f_{3}, f_{4}, f_{5}\right\}$. That is to say all functional units are designated within one module, and all functional tasks appear in an enterprise. This is not conducive to the concurrent design in the dynamic enterprise alliance, and it is generally not adopted.

All the analysis shows that when $\lambda=0.44$, the scheme of tasks Distribution is the best decomposition scheme. In this scheme, it has the largest relative aggregation degree and the relatively small separation degree of the task modules. And it is conducive for the concurrent design and cooperation in the dynamic enterprise alliance.

\section{Conclusions}

This paper presents an optimization method for task granularity at dynamic enterprise alliance layer based on fuzzy aggregation, which can reduce the tasks coupling, interaction frequency and the costs between alliance members. And through improving the parallelism between enterprises, the method can significantly shorten the product development period in the aviation enterprise dynamic alliance.

\section{References}

[1] Mali K, Clustering and its validation in a symbolic framework, Patt Recogn Lett, vol.24:23672376, 2003.

[2] HE Qing, Advance in fuzzy clustering theory and application, FUZZY SYSTEMS AND MATHEMATICS, 12(2)89-94, 1998.

[3] ZHANG Wen-zhang, The utility of modern statistical analysis methods and the application of SPSS, Contemporary Chinese press, 2004.

[4] L. A. Zadeh, Fuzzy sets, Information and Control, (8): 338-353, 1965.

[5] GAO Xin-bo, Fuzzy Cluster Analysis and its Application, Xi'an: xidian university publisher, 2004.

[6] YANG Hai-cheng, LIAO Wen-he, The 3D CAD Technology and Application Based on Knowledge, Beijing: Science Press, 2005. 